\title{
Trends in exercise neuroscience: raising demand for brain fitness
}

\author{
Min Chul Lee', Kyeongho Byun², Ji-Seok Kim³ ${ }^{3}$, Hojun Lee ${ }^{4}$, Kijeong Kim ${ }^{5, *}$ \\ 'Department of Sports Medicine, College of Health Science, CHA University, Pocheon, Korea \\ ${ }^{2}$ Faculty of Health and Sport Sciences, University of Tsukuba, Tsukuba, Japan \\ ${ }^{3}$ Department of Physical Education, College of Education, Gyeongsang National University, Jinju, Korea \\ ${ }^{4}$ School of Sports \& Health Science, College of Arts, Kyungsung University, Busan, Korea \\ ${ }^{5}$ School of Exercise \& Sport Science, College of Natural Sciences, University of Ulsan, Ulsan, Korea
}

Physical exercise is increasingly recognized as an important component in the neuroscience related field. What is the targeting of exercise and what accounts for the exercise's benefits observed in neuroscience? Several types of exercise have been studied in various fields across physiological, psychological, and biochemical experiments of neuroscience. However, more clarity is needed to unveil optimal exercise conditions such as frequency, intensity, type, and time. In this review, we briefly highlight the positive effects of exercise on promoting brain function. Key areas relate to exercise neuroscience are as follow: structural level with synaptic plasticity and neurogenesis, functional level with behavioral development, and molecular level with possible mechanisms that involved in exercise-induced brain plasticity. Overall, we provide the importance of understanding the exercise neuroscience and highlight suggestions for future health research.

Keywords: Exercise, Neuroscience, Brain, Neuroplasticity, Neurogenesis

\section{INTRODUCTION}

Neuroscience is growing scientific area comprising a variety of multidisciplinary investigations that seek to understand the relationship between the body and brain. The brain and its functions might be influenced by several factors such as physical exercise, aging, stress, environment, diet that called brain plasticity. Brain plasticity refers to the capacity of the nervous connection system to change its structural, functional and molecular levels. Brain plasticity include the ability for the brain to recover central nervous system (CNS) after disorders or injuries and to ameliorate the altered structures due to pathologies such as neurodegenerative disease, Alzheimer disease, Parkinson disease, mental illness etc. Especially, physical exercise plays a critical role on this brain plasticity. Exercise offers considerable health benefits to the physiological, psychological, social effects. How does brain plasticity change and what conditions of exercise are applied? This is a highly im- portant issue for non-pharmacological therapeutic and genetic research. That offers the enormous answers, while yet others undergo no change at all or reverse the changing trend. The effects of physical exercise on the structures and functions of the CNS have received increasing attention from the neuroscience. These effects have been applied to studies of the health promotion, exercise science and sports performance. In this review, we provide the recent advances in the beneficial effects of exercise on brain function and highlight sports research.

\section{STRUCTURAL LEVEL WITH SYNAPTIC PLASTICITY AND NEUROGENESIS}

It is well known that physical activity is effective for enhancing both aerobic capacity as well as brain function. A number of studies have shown that exercise-induced improvements in CNS such as synaptic plasticity, and hippocampal neurogenesis is thought to
${ }^{\star}$ Corresponding author: Kijeong Kim (D https://orcid.org/0000-0001-9233-3096 School of Exercise \& Sport Science, College of Natural Sciences, University of Ulsan, 93 Daehak-ro, Nam-gu, Ulsan 44610, Korea

E-mail: kijeongk@ulsan.ac.kr

Received: January 25, 2019 / Accepted: February 17, 2019
This is an Open Access article distributed under the terms of the Creative Commons Attribution Non-Commercial License (http://creativecommons.org/licenses/by-nc/4.0/) which permits unrestricted non-commercial use, distribution, and reproduction in any medium, provided the original work is properly cited. 
play a crucial role in enhancing cognitive functions, including learning and memory (van Praag et al., 1999; Yamada et al., 2018). A report on the processes of synaptic plasticity and neurogenesis have shown that the battery of clinical exercises for brain stimulation designed by brain fitness encourages creation of new synapses and neural circuits able to reorganize function of damaged area with compensatory transmission capabilities by physical exercise (Lourenco et al., 2019). Synaptic plasticity is perhaps the support on which the brain's amazing malleability rests.

Whereas synaptic plasticity is achieved through enhancing communication at the synaptic site between existing neurons, neurogenesis refers to the birth and proliferation of new neurons in the brain throughout whole life (Altman and Das, 1965). When engaged in new experiences and learning, the brain establishes a series of neural pathways. These neural pathways, or circuits, are routes made of interconnecting neurons. Physical exercise enhances hippocampal neurogenesis and spatial memory related to brainderived neurotrophic factor (BDNF) (Sun et al., 2017). These newly generated neurons in a neural pathway communicate with each other through synaptic connections. A better connection between the neurons means that the electric and chemical signals travel more efficiently when creating or using a new pathway. These results suggest that exercise enhances not only structural levels but also functional levels such as learning and memory in individuals.

\section{FUNCTIONAL LEVEL WITH BEHAVIORAL DEVELOPMENT}

The relationships between physical exercise and academic achievement in school-age children, demonstrated significant and positive correlations (Fedewa and Ahn, 2011). In particular, researchers show that brain plasticity is activated and strengthened by applying this clinical intervention exercise program (GomesOsman et al., 2017; Voss et al., 2013). An acute mild exercise improves the executive functions in young adults (Byun et al., 2014). Furthermore higher fitness group had better performance (Suwabe et al., 2017). Also, in the animal studies both wheel and treadmill exercise enhanced animal's performances in Morris water maze (Lee et al., 2012), object recognitions (Robison et al., 2018), Y-maze (Van der Borght et al., 2007), and passive avoidance (Liu et al., 2008) that have been demonstrated on cognition improving in hippocampal associated learning and memory for rodents. Reconsidering the neural circuit and reestablishing neuronal transmission between the implicated neurons at each new attempt enhances the efficiency of synaptic transmission. Communication between the relevant neurons is facilitated, cognition made faster and faster. Functional level with behavioral development seems ideal for inducing brain plasticity. It provides the systematic practice necessary for establishing new neural circuits and for strengthening the synaptic connections among the neurons in the circuit.

\section{MOLECULAR LEVEL WITH POSSIBLE MECHANISMS}

The mechanisms underlying exercise-induced neuroplasticity involve molecular level with several neurotrophic and growth factors. Among them, BDNF is a well-characterized mediator of neuronal growth, plasticity, and survival (Hohn et al., 1990; Vaynman et al., 2003). Human and animal studies collectively suggest that exercise is an active strategy to up-regulate the expression of BDNF, which plays an essential role in exercise-induced neuroplasticity (Cotman and Berchtold, 2002; Neeper et al., 1995; Szuhany et al., 2015). Insulin-like growth factor (IGF)-1 is also a key modulator of neuronal functions in the CNS, including synaptic plasticity, synapse density, neurotransmission, neurogenesis and neuron differentiation (Aleman and Torres-Alemán, 2009). Chronic exercise-enhanced hippocampal neurogenesis, learning and memory performance have been attributed to IGF-1 signaling in the hippocampus (Cetinkaya et al., 2013). Voluntary exercise is known to increase the expressions of vascular endothelial growth factor (VEGF) and its receptors (Stevenson et al., 2018). VEGF, an angiogenic protein, is known to have neuroprotective and neurotrophic functions, it can be synthesized and released by peripheral vascular endothelial cells and brain cells, including astrocytes, ependymal cells, and neuronal stem cells (Nowacka and Obuchowicz, 2012).

Also, nerve growth factor (NGF) plays a role in promoting neuronal function, especially the survival of neural progenitors. The expressions of hippocampal NGF and one of its receptors, tropomyosin receptor kinase A (TrkA), are increased after 8 weeks of treadmill running in rodents. Similar to BDNF/tropomyosin receptor kinase B (TrkB) signaling, the binding of NGF to TrkA stimulates the downstream transcription factor, c-AMP response element binding protein, and induces various gene transcriptions related to cell survival and neuroplasticity (Frielingsdorf et al., 2007). Higher levels of BDNF and TrkB receptor in the hippocampus exhibit better performance in radial water mazes (Alomari et al., 2016) and passive avoidance tasks (Berchtold et al., 2010), blocking the TrkB receptor with antagonist inhibits the efficacy of exercise induced up-regulation of synaptic plasticity related 
proteins significantly, resulting in a disruption of benefits on cognitive function (Ko et al., 2018; Vaynman et al., 2003). All together, exercise seems to be capable of increasing the production of molecular levels in the brain of subjects and the translation of synaptic plasticity related proteins, which finally improves neuroplasticity.

\section{CONCLUSIONS}

Human and animal studies indicate that physical exercise has favorable effects on brain function. Various types of exercise set into motion an interactive cascade of growth factor signaling that has the net effect of stimulating structural changes, enhancing behavioral development and stimulating molecular levels. For example, it is ingenious to note that acute and chronic voluntary resistance wheel running with $30 \%$ of body weight led to greater enhancements, including adult hippocampal neurogenesis and cognitive functions, in conjunction with hippocampal BDNF signaling (Lee et al., 2012; Lee and Soya, 2017). There is no doubt that brain health could be improved through this physical exercise. Exercise benefits neuroplasticity in health and disease stages by targeting different aspects of brain function.

Although, it has been made dramatic accumulation of evidence of exercise neuroscience, there is a need for rigorous clinical intervention trials for further research. Physical, behavioral, and psychosocial barriers limit adequate uptake of exercise and engagement in physical activity. Accordingly, this inconsistency might due to different exercise conditions such as frequency, intensity, type, and time. It needs to identify and to target mechanisms by which exercise might act most synergistically with key treatments

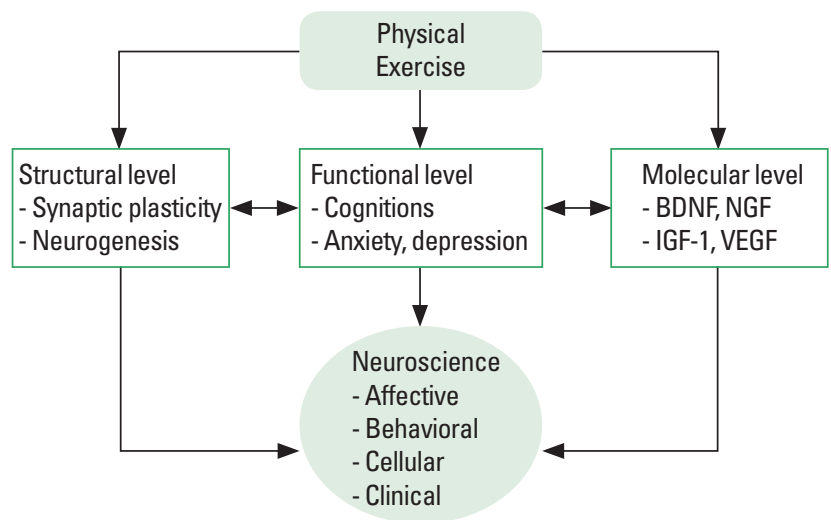

Fig. 1. Exercise-dependent benefits in structural, functional and molecular levels. BDNF, brain-derived neurotrophic factor; NGF, nerve growth factor; IGF-1, insulin-like growth factor -1; VEGF, vascular endothelial growth factor. to improvements. Thus, it should be emphasized that exercise-induced changes in structural, functional and molecular levels may affect performance in both rodent and human (Fig. 1). Overall, studies of the exercise neuroscience aim to explore the effects of physiological and psychological on performance and mental health.

\section{CONFLICT OF INTEREST}

No potential conflict of interest relevant to this article was reported.

\section{ACKNOWLEDGMENTS}

This work supported by the National Research Foundation of Korea Grant funded by the Korean Government (NRF-2017R1C1B5016131).

\section{REFERENCES}

Aleman A, Torres-Alemán I. Circulating insulin-like growth factor I and cognitive function: neuromodulation throughout the lifespan. Prog Neurobiol 2009;89:256-265.

Alomari MA, Khabour OF, Alzoubi KH, Alzubi MA. Combining restricted diet with forced or voluntary exercises improves hippocampal BDNF and cognitive function in rats. Int J Neurosci 2016;126:366-373.

Altman J, Das GD. Autoradiographic and histological evidence of postnatal hippocampal neurogenesis in rats. J Comp Neurol 1965;124:319335.

Berchtold NC, Castello N, Cotman CW. Exercise and time-dependent benefits to learning and memory. Neuroscience 2010;167:588-597.

Byun K, Hyodo K, Suwabe K, Ochi G, Sakairi Y, Kato M, Dan I, Soya H. Positive effect of acute mild exercise on executive function via arousal-related prefrontal activations: an fNIRS study. Neuroimage 2014; 98:336-345.

Cetinkaya C, Sisman AR, Kiray M, Camsari UM, Gencoglu C, Baykara B, Aksu I, Uysal N. Positive effects of aerobic exercise on learning and memory functioning, which correlate with hippocampal IGF-1 increase in adolescent rats. Neurosci Lett 2013;549:177-181.

Cotman CW, Berchtold NC. Exercise: a behavioral intervention to enhance brain health and plasticity. Trends Neurosci 2002;25:295-301.

Fedewa AL, Ahn S. The effects of physical activity and physical fitness on children's achievement and cognitive outcomes: a meta-analysis. Res Q Exerc Sport 2011;82:521-535.

Frielingsdorf H, Simpson DR, Thal LJ, Pizzo DP. Nerve growth factor 
promotes survival of new neurons in the adult hippocampus. Neurobiol Dis 2007;26:47-55.

Gomes-Osman J, Cabral DF, Hinchman C, Jannati A, Morris TP, PascualLeone A. The effects of exercise on cognitive function and brain plasticity - a feasibility trial. Restor Neurol Neurosci 2017;35:547-556.

Hohn A, Leibrock J, Bailey K, Barde YA. Identification and characterization of a novel member of the nerve growth factor/brain-derived neurotrophic factor family. Nature 1990;344:339-341.

Ko IG, Kim SE, Hwang L, Jin JJ, Kim CJ, Kim BK, Kim H. Late starting treadmill exercise improves spatial leaning ability through suppressing CREP/BDNF/TrkB signaling pathway following traumatic brain injury in rats. J Exerc Rehabil 2018;14:327-334.

Lee $\mathrm{M}$, Soya $\mathrm{H}$. Effects of acute voluntary loaded wheel running on BDNF expression in the rat hippocampus. J Exerc Nutrition Biochem 2017; 21:52-57.

Lee MC, Okamoto M, Liu YF, Inoue K, Matsui T, Nogami H, Soya H. Voluntary resistance running with short distance enhances spatial memory related to hippocampal BDNF signaling. J Appl Physiol (1985) 2012;113:1260-1266.

Liu YF, Chen HI, Yu L, Kuo YM, Wu FS, Chuang JI, Liao PC, Jen CJ. Upregulation of hippocampal TrkB and synaptotagmin is involved in treadmill exercise-enhanced aversive memory in mice. Neurobiol Learn Mem 2008;90:81-89.

Lourenco MV, Frozza RL, de Freitas GB, Zhang H, Kincheski GC, Ribeiro FC, Gonçalves RA, Clarke JR, Beckman D, Staniszewski A, Berman H, Guerra LA, Forny-Germano L, Meier S, Wilcock DM, de Souza JM, Alves-Leon S, Prado VF, Prado MAM, Abisambra JF, Tovar-Moll F, Mattos P, Arancio O, Ferreira ST, De Felice FG. Exercise-linked FNDC5/ irisin rescues synaptic plasticity and memory defects in Alzheimer's models. Nat Med 2019;25:165-175.

Neeper SA, Gómez-Pinilla F, Choi J, Cotman C. Exercise and brain neurotrophins. Nature 1995;373:109.

Nowacka MM, Obuchowicz E. Vascular endothelial growth factor (VEGF) and its role in the central nervous system: a new element in the neurotrophic hypothesis of antidepressant drug action. Neuropeptides 2012; 46:1-10.

Robison LS, Popescu DL, Anderson ME, Beigelman SI, Fitzgerald SM,
Kuzmina AE, Lituma DA, Subzwari S, Michaelos M, Anderson BJ, Van Nostrand WE, Robinson JK. The effects of volume versus intensity of long-term voluntary exercise on physiology and behavior in C57/Bl6 mice. Physiol Behav 2018;194:218-232.

Stevenson ME, Behnke VK, Swain RA. Exercise pattern and distance differentially affect hippocampal and cerebellar expression of FLK-1 and FLT-1 receptors in astrocytes and blood vessels. Behav Brain Res 2018; 337:8-16.

Sun L, Sun Q, Qi J. Adult hippocampal neurogenesis: an important target associated with antidepressant effects of exercise. Rev Neurosci 2017; 28:693-703.

Suwabe K, Hyodo K, Byun K, Ochi G, Fukuie T, Shimizu T, Kato M, Yassa MA, Soya H. Aerobic fitness associates with mnemonic discrimination as a mediator of physical activity effects: evidence for memory flexibility in young adults. Sci Rep 2017;7:5140.

Szuhany KL, Bugatti M, Otto MW. A meta-analytic review of the effects of exercise on brain-derived neurotrophic factor. J Psychiatr Res 2015; 60:56-64.

Van der Borght K, Havekes R, Bos T, Eggen BJ, Van der Zee EA. Exercise improves memory acquisition and retrieval in the Y-maze task: relationship with hippocampal neurogenesis. Behav Neurosci 2007;121: 324-334.

van Praag H, Christie BR, Sejnowski TJ, Gage FH. Running enhances neurogenesis, learning, and long-term potentiation in mice. Proc Natl Acad Sci U S A 1999;96:13427-13431.

Vaynman S, Ying Z, Gomez-Pinilla F. Interplay between brain-derived neurotrophic factor and signal transduction modulators in the regulation of the effects of exercise on synaptic-plasticity. Neuroscience 2003; 122:647-657.

Voss MW, Vivar C, Kramer AF, van Praag H. Bridging animal and human models of exercise-induced brain plasticity. Trends Cogn Sci 2013;17: 525-544.

Yamada J, Nadanaka S, Kitagawa H, Takeuchi K, Jinno S. Increased synthesis of chondroitin sulfate proteoglycan promotes adult hippocampal neurogenesis in response to enriched environment. J Neurosci 2018;38:8496-8513. 